\title{
Tensiones entre conservación de ecosistemas y desarrollo territorial: hacia un abordaje socioecológico en las
}

\section{Ciencias Agropecuarias ${ }^{*}$}

Cómo citar este artículo : Easdale, M. H., López, D. R., y Aguiar, M. R. (2018). Tensiones entre conservación de ecosistemas y desarrollo territorial: hacia un abordaje socioecológico en las ciencias agropecuarias. Cuadernos de Desarrollo Rural, I5 (8I), 26-45. https://doi.org/I0.III44/Javeriana.cdri5-8I.tced

Marcos H. Easdale

Instituto Nacional de Tecnología Agropecuaria,

Argentina

easdale.marcos@inta.gob.ar

ORCID: http://orcid.org/0000-0003-1097-5242

Dardo R. López

Instituto Nacional de Tecnología Agropecuaria,

Argentina

ORCID: http://orcid.org/0000-000I-9709-0070

Martín R. Aguiar

Universidad de Buenos Aires, Argentina

ORCID: http://orcid.org/0000-0002-1213-4028

DOI: https://doi.org/10.11144/Javeriana.cdri5-8I.tced

Redalyc: http://www.redalyc.org/articulo.oa?id=11757604002

Recibido: 2I Mayo 20I7 I Publicación: I8 Junio 20I8

\section{Resumen:}

Las ciencias agropecuarias constituyen un vínculo académico entre sociología y ecología. Actualmente, existe la predominancia de una perspectiva tecnocrática orientada a aumentar la productividad y la eficiencia, con una impronta muy operativa e instrumental en el abordaje de muchos problemas complejos, relegando a segundo plano muchos otros aspectos que debieran abordarse en relación con la sustentabilidad y el desarrollo rural. El objetivo de este artículo es discutir el papel de las ciencias agropecuarias como nexo académico para abordar problemáticas socioambientales y la tensión que enfrenta la conservación de los ecosistemas rurales y el desarrollo territorial.

Palabras clave: agronomía, interdisciplina, sistemas socioecológicos, sustentabilidad. 


\title{
Tensions between Ecosystem Preservation and Territorial Development: Towards a Social-Ecological Approach in the Farming Sciences
}

\begin{abstract}
:
The farming sciences are an academic link between sociology and ecology. Currently, there is a prevalent technocratic perspective intended to increase both the productivity and the efficiency with a strong instrumental and operative drive in order to cope with many complex problems. This way some other aspects have been put in the background, even though they should be considered when dealing with the rural development and sustainability. The paper aims to discuss on the role of the farming sciences as an academic link useful to cope with social-environmental problems and the tensions arising in the preservation of rural ecosystems and the territorial development.
\end{abstract}

Keywords: agronomy, interdisciplinary, social-ecological systems, sustainability.

\section{Introducción}

La finalidad de este artículo es discutir el papel de las ciencias agropecuarias en el abordaje de las problemáticas socioambientales y la tensión que enfrenta la integridad y conservación de los ecosistemas rurales y el desarrollo territorial, en el contexto argentino. Por un lado, esta tensión se asocia con una demanda de la economía de mercado por producir de manera sostenida materias primas que ingresan en el circuito económico como camino para contribuir con el crecimiento económico del territorio nacional o continental. Esta vía impulsa la necesidad de generar promoción social y productiva de lo que se conoce como economías regionales, que ocurre de manera asociada al aumento de la productividad y la eficiencia. Pero un desarrollo regional sustentable y equitativo requiere de perspectivas que integren en la gestión las particularidades socioambientales de cada lugar. Por otro lado, es creciente la demanda de la sociedad por evitar la degradación de las funciones del ecosistema (comúnmente referidas a servicios ecosistémicos), pues se percibe que son claves para la sustentabilidad regional y global y, por lo tanto, para el futuro de la humanidad (Dobson et al., 2006; Fischer et al., 2017; Sousa Santos, 20II).

Uno de los principios generales necesarios para abordar este desafío es reconocer que el desarrollo rural sustentable y la gestión ambiental son retos complejos que requieren de la integración de las ciencias sociales y la ecología (Pretty, 20II). Los sistemas sociales están inextricablemente vinculados a sistemas ecológicos. Por ello, cambios en un dominio del sistema (humano o ambiental) inevitablemente producen impactos sobre el otro dominio, debido a su interdependencia (Walker y Salt, 2006). La literatura reciente los denomina sistemas socioecológicos (SSE) o también sistemas humano-ambientales, por lo que hace explícito este vínculo como objeto de estudio (Berkes y Folke, 1998; Gunderson y Holling, 2002). Una de las principales características de los SSE es que no cambian de una manera predecible, lineal, ni de un modo aditivo, por lo que la comprensión del funcionamiento de sus componentes no significa necesariamente que se pueda predecir su comportamiento general (Folke, 2006; Walker, Holling, Carpenter y Kinzig, 2004).

La dimensión sociocultural e institucional de una región, y su asociación con la valoración y gestión de la naturaleza, es también una faceta compleja y de necesaria vinculación entre disciplinas científicas (Brondizio, Ostrom y Young, 2009; Cáceres, Tapella, Quétier y Díaz, 2015). Algunos autores amplían el límite de este concepto 
para incluir el uso tecnológico (agropecuario, por ejemplo) que se hace de los recursos naturales (Smith y Stirling, 20ro). Es aquí donde las ciencias agropecuarias cumplen un papel clave como herramienta para resolver la doble tensión que enfrenta la producción agropecuaria. La intención de este trabajo es problematizar el abordaje dominante en las ciencias agropecuarias, dado que tiene falencias de integración a nivel sistémico, pues su principal aproximación en la generación de conocimiento está asentada en el diagnóstico de partes o componentes de un sistema y justifica, con ello, una posición de objetividad científica. Si bien este abordaje es eficiente para la resolución de problemas particulares (a escala de un lote o potrero, por ejemplo), es muy limitado cuando se pretende intervenir en problemáticas complejas de sistemas socioecológicos (Chapin III, Kofinas y Folke, 2009).

Es frecuente encontrar que se enfatizan relaciones directas de tipo causa-efecto o medidas de gestión de tipo impulso-respuesta. Nuestra hipótesis de trabajo propone que esta segmentación en el estudio del sistema representa un resguardo epistemológico orientado a posicionar la tecnología como un mero instrumento de mejora, basado en la concepción de que el desarrollo tecnológico es resultado de la objetividad científica e imparcialidad política (Aguiar, 2002). El determinismo tecnológico responde a la creencia según la cual la tecnología es capaz, por ella misma, de incidir de manera directa y positiva en el desarrollo socioeconómico de un grupo o en un determinado contexto social. Esta teoría coloca la tecnología en el eje central de los motivos por los que se producen cambios sociales en el transcurso de la historia, por lo cual la considera el factor determinante del progreso y desarrollo social. Dicha teoría asevera que la tecnología es autónoma e independiente de las influencias sociales (Pérez Salazar, 2006). En el contexto agropecuario, consideramos que un nuevo abordaje es necesario para enfrentar los retos que plantea la intervención y gobernanza en procesos de transición o de transformaciones sociotecnológicas (Smith, Stirling y Berkhout, 2005). Este cambio necesita un movimiento hacia una conceptualización de la innovación tecnológica en un modelo productivo y de desarrollo rural explícito que considere particularidades sociales y ambientales.

Avanzar en el abordaje de la complejidad tanto de los problemas como de la organización y la dinámica de los SSE rurales, en pos de la sustentabilidad, requiere de nuevas aproximaciones teóricas y metodológicas que trasciendan las disciplinas tradicionales, las cuales son fuente actual de debate (Anderson et al., 2015; Jansen, 2009; Jerneck et al., 20II; Phillipson, Lowe y Bullock, 2009; Salas-Zapata, Ríos-Osorio y Álvarez-del Castillo, 20I2). A pesar de los profundos cambios acontecidos en la naturaleza y en la sociedad urbana y rural en el último siglo, la organización disciplinaria del conocimiento científico continúa vigente en gran medida sin mayores modificaciones (Metzger y Zare, 1999). Antes de avanzar sobre estos aspectos, creemos conveniente hacer explícito lo que a nuestro criterio es el papel que deberían cumplir las ciencias agropecuarias en el contexto de las problemáticas actuales.

\section{Estado y tendencia de las ciencias agropecuarias en la actualidad}

El punto más relevante en la discusión que proponemos es que en la actualidad existe, en las ciencias agropecuarias de Argentina, la predominancia de una perspectiva tecnológico-productiva ("ingenieril”) orientada a aumentar la productividad por unidad de superficie como una forma de contribuir a la seguridad alimentaria (Godfray et al., 2010). Esta orientación ha ido generando una impronta muy operativa e instrumental en el abordaje de muchos problemas. Por ejemplo, un estudio de mediados de la década de 1990 registró que un $70 \%$ de las publicaciones científicas argentinas en el ámbito agropecuario se concentraban en ejes netamente disciplinarios, como la biología de plantas, la sanidad y la producción vegetal y la biología animal (Oesterheld, Semmartin y Hall, 2002). Una 
de las funciones objetivo que ha dominado la generación de las principales preguntas de investigación ha estado referida al incremento en la eficiencia y la productividad de alimentos, fibras, maderas o biomasa para producción de energía. Estas decisiones, explícitas o implícitas, han circunscripto la discusión de los sistemas agropecuarios al servicio de la productividad, por lo que han obstaculizado los debates en relación con los modelos socioecológicos que buscan optimizar múltiples funciones, tanto sociales (que incluyen los aspectos económicos y culturales) como ambientales (Altieri y Toledo, 20II).

Actualmente, el desarrollo rural se plantea con frecuencia como emergente de una mejora en la eficiencia productiva y de un crecimiento económico regional. Dicho crecimiento es promovido por un sujeto racional, que representa un arquetipo social sin mayores restricciones que las de contar con la capacidad económica para mantenerse actualizado tecnológicamente. Este supuesto tiene como argumento que la tecnología es capaz de incidir de manera directa y positiva en el desarrollo socioeconómico de un grupo o contexto social determinado (Pérez Salazar, 2006). Se ha propuesto que la dominancia de un enfoque tecnológico se puede asociar a un desarrollo rural con visión de empresa capitalista y con fundamentos en el positivismo tecnológico (Vélez Vargas, 2004). Mientras tanto, las facetas más ecológicas y humanas de la agronomía han estado frecuentemente desarticuladas entre sí. Aún se evidencian ejes dominantes (aunque paralelos), como la conservación del suelo, el uso y el manejo del agua, la salud humana en relación con los agroquímicos, lógicas y percepciones de sujetos sociales, estudios de estructura agraria o márgenes económicos de sistemas de producción. Un ejemplo paradigmático es el abordaje de la problemática de la desertificación en Argentina, dominada por estudios de erosión y degradación de suelos, pero con muy baja proporción de estudios socioeconómicos (Torres, Abraham, Rubio, Barbero-Sierra y Ruiz-Pérez, 2015). A ello se suma una falta de perspectiva holística en la búsqueda de soluciones a dicho problema que consideren su complejidad socioecológica (Easdale, 20I6). Esta desarticulación se evidencia y está sustentada en la formación de profesionales. Por ejemplo, en los currículos de grado de la carrera de Ingeniería Agronómica (como expresión de las ciencias agropecuarias en Argentina), las disciplinas aplicadas y las especialidades relacionadas con las producciones vegetal y animal ocupan un mayor espacio curricular que las disciplinas generalistas e integradoras (Comisión Nacional de Evaluación y Acreditación Universitaria [Coneau], 20I4), que quedan asociadas a las ciencias sociales (a la sociología rural, por ejemplo) o a la ecología (como la ecología agraria), y existe mucho menos espacio aún para cátedras de integración. El supuesto detrás de este estado de cosas es que la/el profesional hará el trabajo de integración necesario al gestionar la práctica, pero, en la actualidad, una evidencia de que no ha ocurrido esa integración en la práctica es el gran cambio en el uso del suelo promovido por el avance de la frontera agrícola en Argentina. Este proceso genera conflictos socioambientales asociados a la deforestación, que acarrea problemas de inundaciones en diversas zonas (Barchuk et al., 20ro) y un proceso de salinización de aguas y suelos, por ejemplo, en el Chaco árido (Marchesini, Giménez, Nosetto y Jobbágy, 2017). Estas problemáticas sugieren que la integración entre ciencias sociales y ecología es compleja y requiere de nuevas herramientas conceptuales y operativas.

En este estado de situación, resulta necesario avanzar deliberadamente en la integración conceptual de las ciencias sociales y la ecología, para que puedan consolidar disciplinas o enfoques que aborden los problemas socioambientales planteados, que deberían ser parte integral en la formación en ciencias agropecuarias. Ejemplos de abordajes integradores son la etnobiología (Ladio y Lozada, 2009; Lozada, Ladio y Weigandt, 2006), la agroecología (Altieri y Toledo, 20II; Méndez, Bacon y Cohen, 20I3), el estudio de servicios ecosistémicos y su relación con la producción agraria (Balvanera et al., 20I2; Tittonell, 20I4a) y cambios en el paisaje y uso de la tierra (Bürgi y Russell, 200I). Los problemas de desertificación, desforestación, pérdida de biodiversidad, pérdida de soberanía alimentaria o pobreza tienen, en general, un eje común vinculado con las tensiones que generan el uso que se hace de la tierra, la gobernanza territorial, las formas de distribución de sus riquezas, la apropiación de los servicios ecosistémicos y las externalidades negativas que generan muchas acciones antrópicas y que no son asumidas como pasivos del proceso productivo. Por ejemplo, la agricultura industrial y la deforestación son unas de las principales causantes del incremento de $\mathrm{CO}_{2}$ atmosférico y de la pérdida de la biodiversidad. Si bien existen 
conceptualizaciones que intentan abordar esta situación (Collins et al., 20II), aún no está claro el papel que debieran asumir las disciplinas académicas, en especial las ciencias agropecuarias, respecto a las actividades productivas. Un primer paso es reconocer estas tensiones y la necesidad de superar la dicotomía entre posturas productivistas y conservacionistas de la naturaleza (Tittonell, 2014a), es decir, considerar que si bien existen áreas de sacrificio y áreas necesariamente de conservación de la biodiversidad con reducida intervención humana (como los parques nacionales, por ejemplo), también es una prioridad abordar y consolidar socioecosistemas productivos. Esto se debe a que los socioecosistemas productivos se encuentran en las áreas de mayor extensión espacial, en las cuales el ser humano mantiene una codinámica con la naturaleza y donde la sustentabilidad del uso productivo se debe basar en la concepción de un socioecosistema integrado. En otras palabras, es clave el mantenimiento de una matriz de ecosistemas naturales como sostén de los medios de vida, pues el ecosistema es el hábitat de y provee bienes y servicios al ser humano (Fischer et al., 20I4).

Por otro lado, las investigaciones en torno a la producción de alimentos no pueden estar sujetas solamente al crecimiento económico, a la demanda del mercado o al impacto en el ambiente (Otegui y Oesterheld, 20I3; Semmartin, Bollani, Oesterheld y Benech-Arnold, 20I2); es necesario integrarlas en una conceptualización de la matriz socioproductiva deseable para la sociedad en su conjunto y en términos territoriales (a escala provincial o nacional). Por ejemplo, la soberanía alimentaria es un concepto que destaca la importancia de los sujetos sociales que viven y producen en áreas rurales, sus estilos de producción y la distribución social del uso y la apropiación de los servicios ecosistémicos y de la riqueza generada (Altieri y Toledo, 20II; Borras Jr. y Franco, 2010). Sin embargo, en foros o ámbitos científico-técnicos en donde se tratan temas productivos y agronómicos, se lo considera aún un concepto meramente ideológico (como si la postura produccionista fuera neutra ideológicamente). Es necesario plantearnos qué representa una visión tradicionalista, poco propensa a innovar y que solo involucra cambios positivos si se la asocia con incorporar tecnología basada en subsidios de materia y energía. Esta perspectiva tiene una premisa fuerte, dominada por la necesidad de abastecimiento de alimentos y fibras para una población global creciente y una ascendente demanda de productos agropecuarios (Godfray et al., 2010). El problema de esta premisa es que la producción actual en el mundo ya sería suficiente para proveer las calorías necesarias para alimentar a la población mundial existente, e incluso para la proyectada de nueve a diez mil millones de personas que se espera que existan en el año 2050 (Perfecto y Vandermeer, 2012). Estos dos autores proponen que es importante reconocer que el enfoque en la oferta global creciente de alimentos ignora los conflictos socioambientales y las relaciones de poder, por lo cual un segmento de la población pasa hambre mientras otro, podría decirse, "come demasiado para su propia salud”.

En este sentido, es llamativo que, siendo la actividad agropecuaria la responsable del mayor cambio realizado en el uso de la tierra (a nivel regional y planetario), en el currículo obligatorio de Ingeniería Agronómica en Argentina tampoco se incorporen formalmente materias obligatorias como biogeografía y ordenamiento territorial (Coneau, 20I4). Consideramos que estas disciplinas (o similares) son fundamentales para contemplar integralmente las características biofísicas de un lugar y las tensiones actuales y futuras entre aspectos ambientales, sociales y económico-productivos, con abordajes que integran tanto escalas prediales y regionales como territoriales. Si bien se abordan de forma aislada aspectos de ordenamiento de cuenca y tierras para hacer efectivos el manejo y la conservación de tierras o suelos, estos análisis se basan principalmente en indicadores biofísicos. Estos están asociados a la aptitud productiva o degradación de suelos (tipos de suelo, disponibilidad de agua, fisiografía de cuencas, etcétera), pero en general no se abordan explícitamente los conflictos de uso (como los sectores de la sociedad preocupados por mantener servicios ecosistémicos en general o los sectores rurales detrás del servicio de provisión) y de intereses sectoriales (habitantes rurales versus compañías agroforestales, por ejemplo). Como consecuencia, se mantiene aún un desbalance en la formación de los/as agrónomos/as orientado al manejo y la administración de predios y no al manejo de mosaicos de paisaje o una región, y mucho menos a un abordaje de socioecosistemas que integre diferentes escalas (predial-regional). Por ello, en general, las soluciones propuestas 
son mayoritariamente de tipo tecnológico (Vélez Vargas, 2004), a escala predial o incluso a escala de lote o potrero. Asimismo, es llamativo que se encuentre muy reducida en el currículo la formación en términos de historia y política agraria, generalmente condensadas en materias de extensión rural.

En esencia, el foco de gestión de los asuntos rurales prioriza la situación actual o corriente como escenario dado, obviando que este ha sido determinado por una evolución histórica de cambios biofísicos y pujas de poder manifestadas en decisiones político-administrativas. De manera implícita, lo regional es concebido como un proceso aditivo de unidades menores en la toma de decisiones (como los predios), que no da cuenta de propiedades emergentes al aumentar las escalas espaciales y temporales (movimiento de materiales y organismos) y aún más importante es que no da cuenta de la heterogeneidad propia de la escala de paisaje o la escala regional (Meentemeyer y Box, 1987). En este contexto, las ciencias agropecuarias deben promover nuevos espacios para el desarrollo de marcos teóricos innovadores, adaptados a los cambios actuales y a la complejidad y dinámica de los problemas que enfrenta como ciencia. Por ello, planteamos la necesidad de que las ciencias agropecuarias deben desarrollar su potencial transdisciplinario, integrando distintas disciplinas científicas. Este desarrollo permitirá promover teorías necesarias para impulsar un abordaje acorde con las necesidades actuales, para colaborar en un segundo desafío: acercar nuevos conocimientos científicos a la toma de decisiones que realiza la sociedad.

\section{La necesidad de una nueva agronomía: entre la ingeniería y una ciencia transdisciplinaria}

El enfoque socioecológico propone un espacio que deliberadamente intenta ser convergente, y los sistemas agropecuarios constituyen uno de los vínculos socioecológicos más extendidos en la Tierra. Dicho vínculo se sustenta en la necesidad básica de obtener alimento para la humanidad y la consecuente intervención que se ejerce en la naturaleza para conseguir tal propósito. Con la finalidad de profundizar la discusión y aplicación del enfoque de sistemas socioecológicos (SSE) en las ciencias agropecuarias, se desarrollan a continuación seis ejes conceptuales que podrían contribuir en la construcción de una nueva agronomía: i) aspectos epistemológicos, 2) coevolución, 3) heterogeneidad rural y diversidad agropecuaria, 4) estructuras y funciones socioecológicas, 5) integración de patrones y procesos y 6) propiedades emergentes (vulnerabilidad y resiliencia). Dichos ejes constituyen una lista inicial y surgen como síntesis de una revisión bibliográfica orientada a identificar los pilares fundamentales para el desarrollo de una ciencia transdisciplinaria (Max-Neef, 2005), aplicada en este caso a desarrollar un abordaje socioecológico en las ciencias agropecuarias.

\section{Aspectos epistemológicos del estudio de sistemas socioecológicos}

La formalización de sistemas socioecológicos como objetos de estudio o epistémicos conlleva una transformación en el abordaje, desde una estructuración débil hacia una más robusta, principalmente en términos de la relación 
entre la sociedad y la naturaleza (Becker, 20I2). Una de las distinciones más importantes en las ciencias sociales (y según el principio de incertidumbre de Heisenberg - año 1925- en todas las ciencias) es que el observador no puede despegarse completamente del objeto de estudio, sino que está inmerso en él, dada su condición humana en un contexto social. Por lo tanto, su observación está, en alguna medida y al mismo tiempo, afectando la organización y el funcionamiento del sistema de estudio (Haig, 1995; Phillipson et al., 2009). Un aspecto vinculado y que influye en el abordaje de la dinámica de SSE es que el ser humano, a diferencia del resto de los seres vivos, tiene un comportamiento ético, lo cual implica que sus acciones tienen en cuenta, de una manera comprensiva, el impacto que generan sobre otras (Ayala, 2010). Desde este punto de vista, existen diversas perspectivas y, por lo tanto, definiciones de lo que significa sustentabilidad (Thompson, 2007), con base en múltiples "verdades", valores, experiencias, conocimientos o creencias provenientes de la construcción de la realidad que hace cada individuo inmerso en la sociedad en un momento determinado (Röling, 2003). Pocas veces existe un reconocimiento de que los sistemas agropecuarios son sistemas de actividades construidos por el ser humano y de que, por ende, la comprensión de "lo que es sustentable" va a estar influenciada por valores y sujeta a permanente cambio (Pearson, 2003). Aproximaciones nuevas deberían incluir la perspectiva de diferentes sujetos sociales y sus percepciones y valoraciones asociadas a los estilos de producción y a los beneficios y usos antrópicos de los servicios que brinda la naturaleza (Cáceres et al., 2015). La conceptualización de sustentabilidad debe estar orientada por la multiplicidad de criterios determinados por una sociedad o grupo social que habita un territorio (Pearson, 2003) o, alternativamente, reconocer los valores que subyacen en las consideraciones que se expresan.

\section{Coevolución socioecológica: una mirada a través del tiempo}

Los sistemas socioecológicos se han desarrollado en un proceso coevolutivo entre dinámicas sociales y ambientales (Kallis y Norgaard, 2010). El emergente de esta coevolución está reflejado en las configuraciones y los funcionamientos socioecológicos, adaptados a las condiciones particulares del territorio que lo alberga. En este sentido, los sistemas sociales y ambientales pueden coevolucionar, de manera que muchos ecosistemas naturales presentan paisajes con determinados rasgos ambientales (como heterogeneidad de la vegetación) que han sido modulados por características propias de los sistemas sociales que habitaban o habitan esos ecosistemas, como conocimientos, valores que tienen respecto del ambiente y el tipo y diversidad de organizaciones sociales y tecnologías. Del mismo modo, los sistemas sociales reflejan las características de los sistemas ambientales, como su diversidad social, tasas de productividad, variación espacial y temporal y su resiliencia (Norgaard, I98I). Un ejemplo que ilustra la aplicación del concepto coevolución serían los sistemas ganaderos móviles, en los que el estilo de vida de comunidades nómades y trashumantes se adaptó a los ciclos ambientales y a la heterogeneidad espaciotemporal de una región como estrategia de vida; a la vez, esa dinámica social ha modulado -y modulala heterogeneidad y composición de la vegetación (Easdale, Aguiar y Paz, 2016; Fernández-Giménez y Le Febre, 2006). Por otra parte, un ejemplo de acciones que no necesariamente consideran la coevolución socioecológica como una propiedad que debe ser atendida sería el avance de la frontera agrícola basada en un modelo que se desarrolló en la pampa húmeda hacia regiones áridas y semiáridas adyacentes en Argentina. Este proceso está impulsado por una lógica empresarial, con dueños y administradores de los campos que viven en las ciudades, alejados de la naturaleza, y que por ende se encuentran distanciados de los ciclos ambientales. Es decir, este es un modelo en el cual existe un gran desacople entre las dinámicas sociales y las ambientales (Cáceres, 2015). El avance de la frontera agrícola hacia ecorregiones como el Espinal, el Gran Chaco o el Mato Grosso fue alertado 
tempranamente (Adamoli y Fernández, 1979) y ha generado en la década reciente un sustancial cambio en el uso de la tierra, que involucró la transformación de bosques y pastizales en cultivos agrícolas (Azcuy Ameghino y León, 2005; Volante et al., 2015). Dicho cambio ha promovido procesos de diferenciación social (Cloquell, Albanesi, Nicola, Preda y Propersi, 2005), homogeneizando el paisaje social y productivo, y ha estabilizado la productividad primaria mediante el subsidio con altos niveles de energía o materia (como las tecnologías de insumos). En muchos casos, para resolver problemas de implementación de los cambios necesarios, se extrapolan linealmente lógicas y tecnologías desarrolladas en una región hacia otras en donde los procesos y controles ecológicos y los sistemas de vida y culturales son muy diferentes (Easdale y Domptail, 20I4). En estos contextos, resulta relevante considerar un diálogo entre el conocimiento científico y la memoria biocultural, en tanto sabiduría tradicional propia de una zona o región (Tengö, Brondizio, Elmqvist, Malmer y Spierenburg, 20I4; Toledo y Barrera-Bassols, 2008). Es decir, la agronomía debería reconocerse como una profesión en constante cambio y de ninguna manera estabilizada, pues necesita la integración de diferentes escalas espaciotemporales e incluso de formas de construir conocimiento. Tanto el sistema ambiental como el cultural enfrentan cambios que se afectan mutuamente y que se retroalimentan de manera positiva y negativa, los cuales se encuentran insertos en un sistema de gobernanza que es cambiante y que no ha resuelto cuáles son las prioridades para la sociedad humana a escala planetaria y regional.

\section{Heterogeneidad rural y diversidad agropecuaria: una interfaz entre el ser humano y la naturaleza}

Los estudios de la heterogeneidad y la diversidad de sistemas agropecuarios han sido desarrollados desde diversas perspectivas, especialmente en disciplinas de sociología rural y economía agraria. En general, se busca conocer la diversidad de tipos de productores y pobladores rurales (Ruben y Pender, 2004), sus estrategias de vida y sus estilos o tipos de producción (Evans, 2009; Paz, 2006; Ploeg, 1994), como el tipo y la cantidad de subsidios de energía que utilizan en sus labores culturales. Esto permite avanzar en la comprensión de algunos procesos sociales y productivos e inferir lógicas y comportamientos dentro de un territorio, que en última instancia son los forzantes del uso de recursos naturales. La comprensión de esta diversidad resulta central para el análisis del cambio social y para la práctica de intervención de políticas públicas y de técnicos asesores (Giarraca y Gutiérrez, 1999). Se ha propuesto que las familias rurales y los productores construyen su identidad y su estilo de vida y de producción y que desarrollan sus ideas y conocimientos en un proceso complejo, histórico y de permanente negociación entre el entorno social y ambiental, por percepciones y valoraciones individuales (Ploeg, 1994; Vanclay, Howden, Mesiti y Glyde, 2006). Recientes propuestas avanzan en vincular explícitamente la diversidad de sujetos sociales con sus respectivas valoraciones de los procesos de la naturaleza (Díaz et al., 20II), marcando la necesidad de un camino interdisciplinario en esta dirección. Futuras investigaciones debieran integrar estas perspectivas con aproximaciones al estudio de las relaciones de poder, la capacidad de cambio de diferentes actores sociales vinculados al ámbito rural y sus impactos relativos sobre el ambiente. En este eje es particularmente relevante hacer explícitas las escalas espaciales y temporales de análisis. 


\section{Estructuras y funciones socioecológicas a través de los medios de vida sustentables}

Existe un consenso generalizado respecto a que en sistemas complejos las estructuras y las funciones son características íntimamente interconectadas, y por lo tanto deben ser analizadas integralmente. En nuestro caso, un socioecosistema de vida rural puede ser abordado desde la perspectiva conceptual de los medios de vida y a partir de allí se pueden comprender las estructuras y funciones subyacentes y sus interconexiones (Easdale y López, 20I6). A diferencia de una aproximación basada solamente en los recursos de producción (tierra, capital y trabajo), un medio de vida (o la configuración del acceso a diferentes tipos de capital) se considera a un arreglo de las capacidades, los activos, los procesos y las actividades necesarias para vivir. Un medio de vida es sustentable cuando una unidad rural puede afrontar y recuperarse de perturbaciones o cambios bruscos (dentro de cierto rango) y mantener sus capacidades y activos tanto en el presente como en el futuro, sin degradar la base de sus recursos originales (Scoones, 1998). Un proceso cultural representado por la medida de la diversidad de sujetos sociales y estilos de producción implica, en última instancia, la manera en que una sociedad pone en juego el acceso y uso de diferentes clases de capitales sociales y ambientales.

La esencia del concepto de capital es que se trata de una existencia o un recurso caracterizado por activos tangibles e intangibles que confieren estructura y que poseen la capacidad de producir un flujo de funciones (Ellis, 2000). Desde una perspectiva agropecuaria, ciertas funciones o procesos clave proporcionan la capacidad de autoorganización del sistema, las capacidades de coordinación y estabilidad en el tiempo, mientras que algunos de ellos están involucrados en un proceso de producción (Ekins, Simon, Deutsch, Folke y Groot, 2003). Una clasificación agregada de los medios de vida, de los cuales típicamente se valen las familias rurales o unidades domésticas en un sistema capitalista, son los capitales naturales, el humano, el productivo o de manufactura, el social y el financiero. El capital natural es un subsistema compuesto por activos biofísicos (suelo, agua, diversidad vegetal y animal, etcétera), con sus funciones y procesos (como productividad primaria, ciclado de nutrientes, captura de carbono o interacciones entre especies y sus movimientos) (Daly, 1994). El capital humano, por su parte, comprende todas las individualidades del hogar o la unidad doméstica (la composición de la familia rural, la salud, el conocimiento, los saberes y habilidades, etcétera), con sus respectivas capacidades (de trabajo y de aprendizaje, los procesos de innovación, etcétera), que son importantes para la consecución de cualquier medio de vida. El capital manufacturado comprende los activos bióticos y abióticos involucrados en procesos de producción de bienes (como ganado doméstico, semillas de cultivos, alambrados, maquinarias e infraestructura en general) y que producen un flujo de funciones de producción (como la productividad de granos, carne, fibra, frutos y leña). El capital social se refiere a las redes sociales e instituciones formales y no formales (que representan diferentes tipos de nodos) que promueven diferentes procesos sociales (como la coordinación de acciones individuales y flujos de información asociados a las interacciones entre actores sociales) (Easdale y López, 2016). Finalmente, el capital financiero aglomera los activos económicos o monetarios (o sus equivalentes, es decir, activos en monedas u otras reservas físicas de valor, remesas, bonos, acciones, etcétera), que ofrecen diferentes funciones financieras (como un beneficio neto o tasas de interés o de retorno). Los medios de vida constituyen una aproximación multidimensional de un sistema agropecuario y podrían representar un espacio común para el desarrollo de un lenguaje convergente entre distintas disciplinas científicas, como, por ejemplo, en el estudio y la intervención en procesos de desertificación (Easdale, 20I6). 


\section{Integración de patrones y procesos: perspectiva geográfica y de redes}

El desafío de integrar patrones y procesos en sistemas socioecológicos requiere de un cambio de perspectivas conceptuales y de análisis (Cumming, 20II). Originalmente, una región se describía haciendo énfasis en su heterogeneidad en términos de una serie de atributos estructurales (fisonómico-florísticos, relieve-topografía, demográficos, económicos, etcétera) o funcionales (productividad, régimen de disturbios, natalidad, empleo, etcétera). Por otra parte, se propone que un territorio puede ser descripto en términos de procesos sociales y de conexiones que existen entre puntos o nodos que dan origen a dichos atributos estructurales, como por ejemplo metacomunidades, corredores ecológicos, la conectividad logística o comunicacional o las redes sociales y productivas (Barabási, 2009). El primer enfoque, basado en los atributos con entidad física (descripción de la heterogeneidad espacial), tiene en general una perspectiva espacialmente explícita y asume que lo relevante es la composición y su organización (¿qué es y cómo está distribuido?) y su funcionalidad (¿qué procesos se involucran?) (Díaz y Cabido, 200I; Noss, 1990). El segundo enfoque, basado en redes (descripción de las conexiones entre nodos), asume que la conectividad efectiva es relativamente más importante y realista, y la dimensión de análisis se define en un espacio virtual o relacional que no siempre tiene una representación física explícita (¿qué nodos están conectados entre sí y cómo?) (Newman, 2003). En particular, el análisis de redes permite observar la estructura de conexiones en la que un nodo (un actor, un sitio, un caso o una observación, entre otros) se encuentra involucrado. Por ejemplo, la ganadería trashumante constituye una red socioecológica que promueve interconexión entre ecosistemas fragmentados o geográficamente aislados (Easdale et al., 20I6).

La integración entre dimensiones espaciotemporales y de redes es todavía un reto tanto en estudios ecológicos como sociales (Adams, Faust y Lovasi, 20I2; Bodin y Norberg, 2007; Keitt, Urban y Milne, 1997). Se trata de integrar la perspectiva geográfica de la heterogeneidad espacial, en términos de mosaicos (Reynolds y Wu, I999; Vasseur et al., 2013) o mediada por las características topográficas del paisaje y su conectividad estructural (Brooks, 2003), con la perspectiva de redes en la organización y el funcionamiento de especies, entidades y agentes (Bascompte, 2009; McAllister et al., 2008; Proulx, Promislow y Phillips, 2005). La geografía humana ha estudiado con énfasis el vínculo humano-naturaleza, por ejemplo, en la perspectiva de la ecología cultural (Head y Atchison, 2008). La integración de lo geográfico con lo funcional, y su aplicación desde un abordaje socioecológico, permitiría aportarles nuevas perspectivas a futuros estudios en ciencias agropecuarias, con lo que se aumentaría la robustez de las inferencias que se hacen sobre los sistemas.

\section{Propiedades}

emergentes:

resiliencia

\section{socioecológica}

\section{vulnerabilidad}

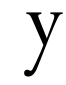

como conceptos

\section{integradores}

La integración de los sistemas biofísicos con los sistemas sociales permite el estudio simultáneo de ciertas propiedades específicas de cada dimensión, pero al mismo tiempo, de propiedades emergentes, como la vulnerabilidad y la resiliencia del socioecosistema. La ciencia de la sustentabilidad se ha desarrollado aceleradamente en las últimas dos décadas (Jerneck et al., 20II), y la vulnerabilidad ha comenzado a ser un 
concepto clave en la sustentabilidad (Gallopín, 2006). El análisis de la vulnerabilidad debe incluir la delimitación y caracterización de un sistema territorial en particular, teniendo en cuenta la identificación de los distintos componentes, las relaciones entre sí y sus vínculos con factores por fuera de los límites del sistema territorial o con procesos que ocurren a distintas escalas espaciotemporales (Smit y Wandel, 2006; Turner II et al., 2003). El análisis de la vulnerabilidad incluye el estudio de las interacciones entre distintas fuentes de cambios abruptos a escalas regionales y globales (como inundaciones, incendios, cambios de precios de bienes agropecuarios, cambios tecnológicos, etcétera) y sus efectos sobre un sistema socioecológico a escala predial (o familiar) y territorial. Por ejemplo, un cambio abrupto, como una inundación, puede resultar en un controlador que beneficia a especies del subsistema natural, pero representar al tiempo una amenaza para los subsistemas humano, productivo o social. En la Depresión del Salado, en la región pampeana argentina, una inundación prologada puede eliminar de los pastizales la mayoría de las malezas y especies exóticas invasoras, pero al mismo tiempo puede provocarles problemas sociales y económicos a los habitantes del territorio, inclusive causando mortalidad de ganado (Insausti, Chaneton y Grimoldi, 2005; Insausti, Chaneton y Soriano, 1999). Es decir, los pulsos de disturbios que son parte de la dinámica del sistema pueden tener efectos divergentes en uno y otro componentes del SSE. Es por ello que para interpretar la vulnerabilidad de un SSE es clave comprender: I) el nivel de exposición del sistema frente a las amenazas (asociado a la dinámica de los factores de disturbio), 2) las características sociales y ecológicas del sistema que determinan su sensibilidad a ser afectado por una o más amenazas a diferentes escalas y 3 ) la resiliencia socioecológica, asociada a las formas o capacidades de respuesta, ajuste y adaptación de un SSE (Easdale y López, 2016; Turner II et al., 2003).

El estudio de la resiliencia en sistemas socioecológicos ha tenido un creciente interés, ya que representa una propiedad emergente clave para la sustentabilidad. Está asociada a interacciones de factores entre escalas, con ciclos de adaptación anidados espacial y temporalmente, que se diferencian de los procesos meramente jerárquicos (Berkes, Colding y Folke, 2003; Berkes y Folke, 1998; Carpenter, Walker, Anderies y Abel, 200I; Chapin III et al., 2009; Folke, 2006; Gunderson y Holling, 2002; López et al., 2017; Walker et al., 2004). La resiliencia ha sido definida originalmente como la capacidad de un sistema de retornar a la condición anterior a la ocurrencia de una perturbación o disturbio, una vez que el factor de disturbio ha sido suprimido o desaparece (resiliencia ecosistémica) (Holling, 1973). Algunas propuestas expanden la definición original de resiliencia para incluir dinámicas sociales y la toma de decisiones (Domptail, Easdale y Yuerlita, 2013; Reyes y Ballesteros, 20II; Walker, Anderies, Kinzig y Ryan, 2006; Walker y Salt, 2006) o sus implicancias en sistemas agropecuarios (Darnhofer, Fairweather y Moller, 20го; Milestad y Darnhofer, 2003; Tittonell, 20I4b). Aplicada a sistemas socioecológicos, la resiliencia se asocia a las capacidades i) de respuesta de un sistema a factores de disturbio, 2) de autorregulación o autoorganización luego de un disturbio y 3) de aprendizaje y adaptación a cambios futuros (Folke et al., 2002; Nelson, Adger y Brown, 2007). Un aspecto relevante en términos operativos es que existe un consenso generalizado en considerar que la diversidad funcional está positivamente relacionada con la resiliencia (Allison, 2004; Mumby, Chollett, Bozec y Wolff, 20I4; Niehof, 2004). Por lo tanto, una menor vulnerabilidad del territorio a diferentes amenazas se asociaría a una mayor diversidad socioecológica y a una mayor resiliencia del SSE (López et al., 2017; Smit y Wandel, 2006; Turner II et al., 2003). En efecto, proponemos que un enfoque socioecológico en los estudios en el ámbito agropecuario debieran articular de manera complementaria y equitativa las perspectivas basadas en el aumento de la productividad y la eficiencia y las perspectivas basadas en la vulnerabilidad y la resiliencia socioecológica. 


\section{A manera de síntesis}

Nuestro ensayo considera que los sistemas agropecuarios constituyen una interfaz entre dinámicas sociales y ecológicas y que son uno de los usos de la tierra más extendidos en el mundo. Dada la complejidad y la dinámica temporal y espacial propia del sistema, es necesario que la agronomía utilice y desarrolle la potencialidad que ofrece su carácter intrínsecamente transdisciplinario. El acercamiento entre disciplinas requiere de un mayor esfuerzo por entender recíprocamente algunas bases filosóficas y epistemológicas (Bernard, 2006; Jansen, 2009), características y restricciones de metodologías utilizadas y los tipos de datos asociados (Bernard, 2006; Cooke et al., 2009; Graymore, Sipe y Rickson, 2008; Madsen y Adriansen, 2004). Esto incluye el reconocimiento de la existencia de problemas de comunicación entre disciplinas y de diferencias en el lenguaje (Fox et al., 2006), que todavía deben ser superados. Las ciencias agropecuarias debieran emprender un nuevo proceso de desarrollo científico para constituir una interfaz transdisciplinaria en el estudio de la sustentabilidad en la relación entre el ser humano y la naturaleza. A su vez, esta podría contribuir a retomar la iniciativa (censurada) que supo tener la ciencia latinoamericana en esta materia en el siglo pasado (Herrera et al., 1977).

Si aceptamos dicha premisa, tomando al contexto argentino como caso inicial de debate (potencialmente aplicable a otros países y zonas de Latinoamérica), proponemos que las ciencias agropecuarias deben complementar su ámbito de comodidad tecnocrática con una perspectiva que permita acoplar el desarrollo tecnológico con los debates sobre los modelos productivos y sus implicancias sociales y ambientales. Consideramos que los seis ejes propuestos sintetizan conceptos y desafíos claves, que integran consideraciones epistemológicas y de abordaje de la complejidad del nuevo sistema que sería objeto de estudio. Estos elementos constituyen una primera base para futuros desarrollos en el estudio, la gestión y la gobernanza de sistemas socioecológicos agropecuarios.

Finalmente, este camino necesariamente requiere hacer más explícitos los objetivos reales de los actores y, al mismo tiempo, los valores y las posturas ideológicas que subyacen a las investigaciones y acciones en ámbitos rurales. Estos deberían formar parte de la discusión de los diseños de los estudios, ya que inciden en la búsqueda de consensos y acuerdos sociales en la relación entre el hombre y la naturaleza, en estos casos mediados por procesos de producción agropecuaria.

\section{Agradecimientos}

El presente trabajo tuvo el apoyo del INTA (PRET i28II03) y del Ministerio de Ciencia, Tecnología e Innovación Productiva de Argentina (PICT 2015-929). Agradecemos a los evaluadores anónimos y al equipo editorial por sus valiosas contribuciones, que mejoraron sensiblemente este artículo. Martín R. Aguiar agradece a William Blake por "May God us keep from single vision and Newton's sleep” y a Joni Mitchell por “Oh let your little light shine... on science with its tunnel vision”.

\section{Referencias}

Adamoli, J., y Fernández, P. (1979). Expansión de la frontera agropecuaria en la Cuenca del Plata: antecedentes ecológicos y socio-económicos para su planificación. Chile: Cepal.

Adams, J., Faust, K., y Lovasi, G. S. (20I2). Capturing context: Integrating spatial and social network analyses. Social Networks, 34(I), I-5. https://doi.org/I0.I0I6/j.socnet.20II.I0.007 
Aguiar, D. S. (2002). Determinismo tecnológico versus determinismo social: aportes metodológicos y teóricos de la filosofía, la historia, la economía y la sociología de la tecnología (Tesis de Licenciatura en Sociología). Buenos Aires, Universidad Nacional de La Plata.

Allison, G. (2004). The influence of species diversity and stress intensity on community resistance and resilience. Ecological Monographs, 74(I), II7-134. https://doi.org/I0.I890/02-068I

Altieri, M. A., y Toledo, V. M. (20II). The agroecological revolution in Latin America: Rescuing nature, ensuring food sovereignty and empowering peasants. The Journal of Peasant Studies, 38(3), 587-612. https:// doi.org/10.1080/03066150.2011.582947

Anderson, C. B., Pizarro, J. C., Estévez, R., Sapoznikow, A., Pauchard, A., Barbosa, O., ... Valenzuela, A. E. J. (2015). ¿Estamos avanzando hacía una socio-ecología? Reflexiones sobre la integración de las dimensiones "humanas" en la ecología en el sur de América. Ecología Austral, 25(3), 263-272. Recuperado de http://ojs.e cologiaaustral.com.ar/index.php/Ecologia_Austral/article/view/94

Ayala, F. J. (2010). The difference of being human: Morality. Proceedings of the Natural Academy of Sciences USA, 107(2), 9015-9022. Recuperado de http://www.pnas.org/content/pnas/ro7/Supplement_2/9015.full.pdf

Azcuy Ameghino, E., y León, C. A. (2005). La "sojización": contradicciones, intereses y debates. Revista Interdisciplinaria de Estudios Agrarios, (23), 133-157. Recuperado de http://bibliotecadigital.econ.uba.ar/do wnload/riea/riea_v23_ni_05.pdf

Balvanera, P., Uriarte, M., Almeida-Leñero, L., Altesor, A., DeClerck, F., Gardner, T., ... Vallejos, M. (2012). Ecosystem services research in Latin America: The state of the art. Ecosystem Services, 2, 56-70. https:// doi.org/I0.I0I6/j.ecoser.20I2.09.006

Barabási, A.-L. (2009). Scale-free networks: A decade and beyond. Science, 325(5939), 412-413. Recuperado de http:// science.sciencemag.org/content/325/5939/412

Barchuk, A. H., Barri, F., Britos, A. H., Cabido, M., Fernández, J., y Tamburini, D. (2010). Diagnóstico y perspectivas de los bosques en Córdoba. Revista Hoy la Universidad, (4), 52-73. Recuperado de https://es.sl ideshare.net/navarroj58/diagnstico-y-perspectivas-de-los-bosques-en-crdoba

Bascompte, J. (2009). Disentangling the web of life. Science, 325(5939), 416-419. Recuperado de http://science.sci encemag.org/content/325/5939/416

Becker, E. (2012). Social-ecological systems as epistemic objects. En Human-nature interactions in the Anthropocene: Potentials of social-ecological systems analysis (pp. 37-59). Londres: Routledge.

Berkes, F., Colding, J., y Folke, C. (eds.). (2003). Navigating social-ecological systems: Building resilience for complexity and change. Cambridge, Reino Unido: Cambridge University Press.

Berkes, F., y Folke, C. (eds.). (1998). Linking social and ecological systems: Management practices and social mechanism for building resilience. Cambridge, RU: Cambridge University Press.

Bernard, H. R. (2006). Research methods in anthropology: Qualitative and quantitative approaches (4.a ed.). Reino Unido: Altamira Press.

Bodin, Ö., y Norberg, J. (2007). A network approach for analyzing spatially structured populations in fragmented landscape. Landscape Ecology, 22(I), 31-44. https://doi.org/I0.1007/siog80-006-9015-0

Borras Jr., S., y Franco, J. (2010). Contemporary discourses and contestations around pro-poor land policies and land governance. Journal of Agrarian Change, Io(I), I-32. Recuperado de https://www.tni.org/files/ propoorlandpolicydebates.pdf 
Brondizio, E. S., Ostrom, E., y Young, O. R. (2009). Connectivity and the governance of multilevel socialecological systems: The role of social capital. Annual Review of Environment and Resources, 34, 253-278. http://dx.doi.org/I0.II46/annurev.environ.020708.100707

Brooks, C. P. (2003). A scalar analysis of landscape connectivity. Oikos, I02(2), 433-439. https://doi.org/10.1034/ j.1600-0579.2003.11511.x

Bürgi, M., y Russell, E. W. (200I). Integrative methods to study landscape changes. Land Use Policy, I8(I), 9-I6. https://doi.org/I0.10I6/So264-8377(00)0004I-7

Cáceres, D. M. (2015). Accumulation by dispossession and socio-environmental conflicts caused by the expansion of agribusiness in Argentina. Journal of Agrarian Change, I5(I), I16-I47. https://doi.org/10.IIII/joac.I2057

Cáceres, D. M., Tapella, E., Quétier, F., y Díaz, S. (2015). The social value of biodiversity and ecosystem services from the perspectives of different social actors. Ecology and Society, 20(I), art. 62. http://dx.doi.org/10.575I/ ES-07297-200162

Carpenter, S., Walker, B., Anderies, J., y Abel, N. (200I). From metaphor to measurement: Resilience of what to what? Ecosystems, 4(8), 765-78I. https://doi.org/I0.1007/sio02I-00I-0045-9

Chapin III, F. S., Kofinas, G. P., y Folke, C. (eds.). (2009). Principles of ecosystem stewardship: Resilience-based natural resource management in a changing world. Estados Unidos: Springer.

Cloquell, S., Albanesi, R., Nicola, M. de, Preda, G., y Propersi, P. (2005). La agricultura a escala y los procesos de diferenciación social. Revista Interdisciplinaria de Estudios Agrarios, 23, 35-57. Recuperado de http://bibli otecadigital.econ.uba.ar/download/riea/riea_v23_ni_02.pdf

Collins, S. L., Carpenter, S. R., Swinton, S. M., Orenstein, D. E., Childers, D. L., Gragson, T. L., ,.. Whitmer, A. C. (20II). An integrated conceptual framework for long-term social-ecological research. Frontiers in Ecology and the Environment, 9(6), 351-357. https://doi.org/10.1890/100068

Comisión Nacional de Evaluación y Acreditación Universitaria [Coneau]. (20I4). Acreditación de carreras de grado: Ingeniería Agronómica, impacto en la calidad educativa. Buenos Aires: autor. Recuperado de http:/ /www.coneau.gov.ar/archivos/publicaciones/documentos/AcreditacionCarrerasGrado-Agronomia.pdf

Cooke, I. R., Queenborough, S. A., Mattison, H. A., Bailey, A. P., Sandars, D. L., Graves, A. R., ... Sutherland, W. J. (2009). Integrating socio-economics and ecology: A taxonomy of quantitative methods and a review of their use in agro-ecology. Journal of Applied Ecology, 46(2), 269-277. https://doi.org/10.11II/j.1365-2664.2009.01615.x

Cumming, G. S. (201I). Spatial resilience: Integrating landscape ecology, resilience, and sustainability. Landscape Ecology, 26(7), 899-909. https://doi.org/10.1007/s10980-0II-9623-I

Daly, H. E. (1994). Operationalizing sustainable development by investing in natural capital. En A. M. Jansson, M. Hammer, C. Folke y R. Costanza (eds.), Investing in natural capital: The ecological economics approach to sustainability (pp. 22-37). Washington: Island Press.

Darnhofer, I., Fairweather, J., y Moller, H. (2010). Assessing a farm's sustainability: Insights from resilience thinking. International Journal of Agricultural Sustainability, 8(3), I86-198. https://doi.org/10.3763/ ijas.2010.0480

Díaz, S., Quétier, F., Cáceres, D. M., Trainor, S. F., Pérez-Harguindeguy, N., Bret-Harte, M. S., ... Poorter, L. (20II). Linking functional diversity and social actor strategies in a framework for interdisciplinary analysis of nature's benefits to society. Proceedings of the National Academy of Sciences, 108(3), 895-902. https:// doi.org/10.1073/pnas.1017993108

Díaz, S., y Cabido, M. (200I). Vive la différence: plant functional diversity matters to ecosystem processes. Trends in Ecology \& Evolution, I6(II), 646-655. https://doi.org/I0.1016/Sor69-5347(OI)02283-2 
Dobson, A., Lodge, D., Alder, J., Cumming, G. S., Keymer, J., McGlade, J., ... Xenopoulos, M. A. (2006). Habitat loss, trophic collapse, and the decline of ecosystem services. Ecology, 87(8), 1915-1924. https:// doi.org/10.1890/0012-9658(2006)87[1915:HLTCAT]2.0.CO;2

Domptail, S. E., Easdale, M. H., y Yuerlita. (2013). Managing socio-ecological systems to achieve sustainability: A study of resilience and robustness. Environmental Policy and Governance, 23(I), 30-45. https:// doi.org/10.1002/eet.1604

Easdale, M. H. (2016). Zero net livelihood degradation: The quest for a multidimensional protocol to combat desertification. Soil, 2, I29-134. https://doi.org/10.5194/soil-2-129-20I6

Easdale, M. H., Aguiar, M. R., y Paz, R. (2016). A social-ecological network analysis of argentinean Andes transhumant pastoralism. Regional Environmental Change, 16(8), 2243-2252. https://doi.org/10.1007/ SIOII3-0I5-0917-8

Easdale, M. H., y Domptail, S. E. (20I4). Fate can be changed! Arid rangelands in a globalizing world- a complementary co-evolutionary perspective on the current 'desert syndrome'. Journal of Arid Environments, I00-IOI, 52-62. https://doi.org/I0.I0I6/j.jaridenv.2013.10.009

Easdale, M. H., y López, D. R. (2016). Sustainable livelihoods approach through the lens of the state-and-transition model in semi-arid pastoral systems. The Rangeland Journal, 38(6), 54I-55I. https://doi.org/10.107I/RJi509I

Ekins, P., Simon, S., Deutsch, L., Folke, C., y Groot, R. de. (2003). A framework for the practical application of the concepts of critical natural capital and strong sustainability. Ecological Economics, 44(2-3), 165-185. https:// doi.org/10.1016/S0921-8009(02)00272-0

Ellis, F. (2000). Rural livelihoods and diversity in developing countries. Oxford: Oxford University Press.

Evans, N. (2009). Adjustments strategies revisited: Agricultural change in the Welsh Marches. Journal of Rural Studies, 25(2), 217-230. https://doi.org/10.1016/j.jrurstud.2008.10.002

Fernández-Giménez, M. E., y Le Febre, S. (2006). Mobility in pastoral systems: Dynamic flux or downward trend? International Journal of Sustainable Development \& World Ecology, 13(5), I-22. https:// doi.org/10.1080/13504500609469685

Fischer, J., Abson, D. J., Bergsten, A., Collier, N. F., Dorresteijnm, I., Hanspach, J., ... Senbeta, F. (20I7). Reframing the food-biodiversity challenge. Trends in Ecology \& Evolution, 32(5), 335-345. https://doi.org/10.1016/ j.tree.2017.02.009

Fischer, J., Abson, D. J., Butsic, V., Chappell, M. J., Ekroos, J., Hanspach, J., ... Wehrden, H. (20I4). Land sparing versus land sharing: Moving forward. Conservation Letters, 7(3), I49-157. https://doi.org/I0.IIII/conl.I2084

Folke, C. (2006). Resilience: The emergence of a perspective for social-ecological systems analyses. Global Environmental Change, 16(3), 253-267. https://doi.org/10.1016/j.gloenvcha.2006.04.002

Folke, C., Carpenter, S., Elmqvist, T., Gunderson, L., Holling, C. S., y Walker, B. H. (2002). Resilience and sustainable development: Building adaptive capacity in a world of transformation. Ambio, 3I(5), 437-440. Recuperado de http://citeseerx.ist.psu.edu/viewdoc/download?doi=I0.I.I.629.3759\&rep=repi\&type=pdf

Fox, H. E., Christian, C., Nordby, C., Pergams, O. R. W., Peterson, G. D., y Pyke, C. R. (2006). Perceived barriers to integrating social science and conservation. Conservation Biology, 20(6), I817-1820. https://doi.org/I0.IIII/ j.1523-1739.2006.00598.x

Gallopín, G. C. (2006). Linkages between vulnerability, resilience and adaptive capacity. Global Environmental Change, 16(3), 293-303. https://doi.org/10.1016/j.gloenvcha.2006.02.004 
Giarraca, N., y Gutiérrez, P. (1999). Una aproximación a los estudios agrarios en Europa y en Estados Unidos en este siglo. En N. Giarraca (coord.), Estudios rurales: teorías, problemas y estrategias metodológicas (pp. 53-73). Buenos Aires: La Colmena, Clacso.

Godfray, H. C. J., Beddington, J. R., Crute, I. R., Haddad, L., Lawrence, D., Muir, J. F., ... Toulmin, C. (20I0). Food security: The challenge of feeding 9 billion people. Science, 327(5967), 812-818. Recuperado de http:// science.sciencemag.org/content/327/5967/812

Graymore, M. L. M., Sipe, N. G., y Rickson, R. E. (2008). Regional sustainability: How useful are current tools of sustainability assessment at the regional scale? Ecological Economics, 67(3), 362-372. https://doi.org/I0.1016/ j.ecolecon.2008.06.002

Gunderson, L. H., y Holling, C. S. (eds.). (2002). Panarchy: Understanding transformations in human and natural systems. Washington: Island Press.

Haig, B. D. (1995). Grounded theory as scientific method. Philosophy of Education, 28, I-II. Recuperado de https ://pdfs.semanticscholar.org/5886/43f9dedi59acc42daeefed6fidi952bea546.pdf

Head, L., y Atchison, J. (2008). Cultural ecology: Emerging human-plant geographies. Progress in Human Geography, 33(2), 236-245. https://doi.org/10.1177/0309132508094075

Herrera, A. O., Scolnick, H. D., Chichilnisky, G., Gallopín, C. G., Hardoy, J. E., Mosovich, D., ... Talavera, L. (1977). ¿Catástrofe o nueva sociedad? Modelo mundial Latinoamericano. Ottawa: Centro Internacional de Investigaciones para el Desarrollo.

Holling, C. S. (1973). Resilience and stability of ecological systems. Annual Review of Ecology and Systematics, 4, I-23. https://doi.org/I0.II46/annurev.es.04.110173.000245

Insausti, P., Chaneton, E. J., y Grimoldi, A. A. (2005). Las inundaciones modifican la estructura y dinámica de la vegetación en los pastizales de la Pampa Deprimida. En M. Oesterheld, M. R. Aguiar, C. M. Ghersa y J. M. Paruelo (eds.), La heterogeneidad de la vegetación de los agroecosistemas: un homenaje a Rolando León (pp. 253-269). Buenos Aires: Editorial Facultad de Agronomía.

Insausti, P., Chaneton, E. J., y Soriano, A. (1999). Flooding reverted grazing effects on plant community structure in Mesocosms of lowland grassland. Oikos, 84(2), 266-276. Recuperado de https://www.jstor.org/stable/354 6721?seq=I\#page_scan_tab_contents

Jansen, K. (2009). Implicit sociology, interdisciplinarity and systems theories in agricultural science. Sociologia Ruralis, 49(2), I72-I88. https://doi.org/I0.IIII/j.I467-9523.2009.00486.x

Jerneck, A., Olsson, L., Ness, B., Anderberg, S., Baier, M., Clark, E., ... Persson, J. (20II). Structuring sustainability science. Sustainability Science, 6(I), 69-82. https://doi.org/10.1007/sII625-010-0117-x

Kallis, G., y Norgaard, R. B. (2010). Coevolutionary ecological economics. Ecological Economics, 69(4), 690-699. https://doi.org/10.1016/j.ecolecon.2009.09.017

Keitt, T. H., Urban, D. L., y Milne, B. T. (1997). Detecting critical scales in fragmented landscapes. Ecology and Society, I(I), art. 4. Recuperado de https://www.ecologyandsociety.org/volı/issı/art4/

Ladio, A. H., y Lozada, M. (2009). Human ecology, ethnobotany and traditional practices in rural populations inhabiting the Monte region: Resilience and ecological knowledge. Journal of Arid Environments, 73(2), 222-227. https://doi.org/10.1016/j.jaridenv.2008.02.006

López, D. R., Cavallero, L., Easdale, M. H., Carranza, C. H., Ledesma, M., y Peri, P. L. (2017). Resilience management at the landscape level: An approach to tackling social-ecological vulnerability of agroforestry systems. En F. Montagnini (ed.), Integrating landscapes: Agroforestry for biodiversity conservation and food sovereignty. Estados Unidos: Springer. Advances in Agroforestry, I2. 
Lozada, M., Ladio, A., y Weigandt, M. (2006). Cultural transmission of ethnobotanical knowledge in a rural community of northwestern Patagonia, Argentina. Economic Botany, 60(4), 374-385. https:// doi.org/10.1663/0013-000I(2006)60[374:CTOEKI]2.0.CO;2

Madsen, L. M., y Adriansen, H. K. (2004). Understanding the use of rural space: The need for multi-methods. Journal of Rural Studies, 20(4), 485-497. https://doi.org/10.1016/j.jrurstud.2003.12.005

Marchesini, V. A., Giménez, R., Nosetto, M. D., y Jobbágy, E. G. (2017). Ecohydrological transformation in the Dry Chaco and the risk of dryland salinity: Following Australia’s footsteps? Ecohydrology, ro(4). https:// doi.org/10.1002/eco.1822

Max-Neef, M. A. (2005). Foundations of transdisciplinarity. Ecological Economics, 53(I), 5-I6. https:// doi.org/I0.I0I6/j.ecolecon.2005.0I.0I4

McAllister, R. R. J., Cheers, B., Darbas, T., Davies, J., Richards, C., Robinson, C. J., ... Maru, Y. T. (2008). Social networks in arid Australia: A review of concepts and evidence. The Rangeland Journal, 30(I), 167-176. https:// doi.org/10.107I/RJ07040

Meentemeyer, V., y Box, E. O. (1987). Scale effects in landscape studies. En M. G. Turner (ed.), Landscape heterogeneity and disturbance (15-34). Nueva York: Springer. Ecological Studies, 64. https:// doi.org/10.1007/978-I-4612-4742-5_2

Méndez, V. E., Bacon, C. M., y Cohen, R. (2013). Agroecology as a transdisciplinary, participatory and actionoriented approach. Agroecology and Sustainable Food Systems, 37(I), 3-18. Recuperado de https://www.tan dfonline.com/doi/abs/10.1080/10440046.2012.736926

Metzger, N., y Zare, R. N. (1999). Interdisciplinary research: From belief to reality. Science, 283(5402), 642-643. Recuperado de http://science.sciencemag.org/content/283/5402/642

Milestad, R., y Darnhofer, I. (2003). Building farm resilience: The prospects and challenges of organic farming. Journal of Sustainable Agriculture, 22(3), 8I-97. https://doi.org/10.1300/J064V22n03_09

Mumby, P. J., Chollett, I., Bozec, Y.-M., y Wolff, N. H. (2014). Ecological resilience, robustness and vulnerability: How do these concepts benefit ecosystem management? Current Opinion in Environmental Sustainability, 7, 22-27. https://doi.org/10.1016/j.cosust.2013.11.021

Nelson, D. R., Adger, W. N., y Brown, K. (2007). Adaptation to environmental change: Contributions of a resilience framework. Annual Review of Environment and Resources, 32, 395-419. https://doi.org/10.II46/ annurev.energy.32.051807.090348

Newman, M. E. J. (2003). The structure and function of complex networks. SIAM Review, 45(2), 167-256. https:// doi.org/I0.1137/So036r4450342480

Niehof, A. (2004). The significance of diversification for rural livelihood systems. Food Policy, 29(4), 321-338. https://doi.org/10.1016/j.foodpol.2004.07.009

Norgaard, R. B. (198I). Sociosystem and ecosystem coevolution in the Amazon. Journal of Environmental Economics and Management, 8(3), 238-254. https://doi.org/10.1016/0095-0696(8I)90039-5

Noss, R. F. (1990). Indicators for monitoring biodiversity: A hierarchical approach. Conservation Biology, 4(4), 355-364. https://doi.org/I0.11II/j.1523-1739.1990.tbo0309.x

Oesterheld, M., Semmartin, M., y Hall, A. (2002). Análisis bibliográfico de la investigación agronómica en la Argentina. Ciencia Hoy, I2(70), 52-62. Recuperado de https://www.agro.uba.ar/users/semmarti/papers\%20 pdfs/agronomia\%2ociencia\%2ohoy.pdf

Otegui, M. E., y Oesterheld, M. (2013). Algunos desafíos de las ciencias agropecuarias. Ciencia Hoy, (135). Recuperado de http://cienciahoy.org.ar/2013/10/algunos-desafios-para-las-ciencias-agropecuarias/ 
Paz, R. (2006). El campesinado en el agro argentino: ¿repensando el debate teórico o un intento de reconceptualización? European Review of Latin American and Caribbean Studies, (8I), 65-85. https:// doi.org/10.18352/erlacs.9648

Pearson, C. J. (2003). Sustainability: Perceptions of problems and progress of the paradigm. International Journal of Agricultural Sustainability, I(I), 3-13. https://doi.org/10.3763/ijas.2003.0102

Pérez Salazar, M. C. G. (2006). El determinismo tecnológico: una política de Estado. Revista Digital Universitaria, 7(ı), 2-7. Recuperado de http://www.revista.unam.mx/vol.7/numı/art87/oct_art87.pdf

Perfecto, I., y Vandermeer, J. (20I2). Separación o integración para la conservación de biodiversidad: la ideología detrás del debate land sharing frente a land sparing. Ecosistemas, 2I(I-2), I80-I9I. Recuperado de https://ww w.revistaecosistemas.net/index.php/ecosistemas/article/view/36

Phillipson, J., Lowe, P., y Bullock, J. M. (2009). Navigating the social sciences: Interdisciplinarity and ecology. Journal of Applied Ecology, 46(2), 26I-264. https://doi.org/10.IIII/j.1365-2664.2009.01625.x

Ploeg, J. D. van der. (1994). Styles of farming: An introductory note on concepts and methodology. En J. D. van der Ploeg y A. Long (eds.), Born from within: Practice and perspectives of endogenous rural development. Países Bajos: Van Gorcum.

Pretty, J. (20II). Interdisciplinary progress in approaches to address social-ecological and ecocultural systems. Environmental Conservation, 38(2), 127-139. https://doi.org/10.1017/S0376892910000937

Proulx, S. R., Promislow, D. E. L., y Phillips, P. C. (2005). Network thinking in ecology and evolution. Trends in Ecology and Evolution, 20(6), 345-353. https://doi.org/10.1016/j.tree.2005.04.004

Reyes, J. E., y Ballesteros, E. R. (20II). Resiliencia socioecológica: aportaciones y retos desde la antropología. Revista de Antropología Social, 20, 109-135. Recuperado de http://revistas.ucm.es/index.php/RASO/artic le/view/36264/35113

Reynolds, J. F., y Wu, J. (1999). Do landscape structural and functional units exist? En J. D. Tenhunen y P. Kabat (eds.), Integrating hydrology, ecosystem dynamics and biogeochemistry in complex landscapes. Estados Unidos: John Wiley \& Sons.

Röling, N. (2003). From causes to reasons: The human dimension of agricultural sustainability. International Journal of Agricultural Sustainability, I(I), 73-88. https://doi.org/10.3763/ijas.2003.0108

Ruben, R., y Pender, J. (2004). Rural diversity and heterogeneity in less-favoured areas: The quest for policy targeting. Food Policy, 29(4), 303-320. https://doi.org/10.1016/j.foodpol.2004.07.004

Salas-Zapata, W. A., Ríos-Osorio, L. A., y Álvarez-del Castillo, J. (2012). Marco conceptual para entender la sustentabilidad de los sistemas socioecológicos. Ecología Austral, 22(I), 74-79. Recuperado de http://www.s cielo.org.ar/scielo.php?script=sci_arttext\&pid=SI667-782X2012000100008

Scoones, I. (1998). Sustainable rural livelihoods: A framework for analysis. Institute of Development Studies, IDS Working Paper n.o 72.

Semmartin, M., Bollani, M. V., Oesterheld, M., y Benech-Arnold, R. (20I2). Las ciencias agropecuarias como herramienta de crecimiento económico: un análisis en cuatro cultivos extensivos. Interciencia, 37(6), 457-463.

Smit, B., y Wandel, J. (2006). Adaptation, adaptive capacity and vulnerability. Global Environmental Change, I6(3), 282-292. https://doi.org/10.1016/j.gloenvcha.2006.03.008

Smith, A., Stirling, A., y Berkhout, F. (2005). The governance of sustainable sociotechnical transitions. Research Policy, 34(I0), I49I-1510. https://doi.org/I0.1016/j.respol.2005.07.005 
Smith, A., y Stirling, A. (2010). The politics of social-ecological resilience and sustainable socio-technical transitions. Ecology and Society, I5(I), art. II. Recuperado de https://www.ecologyandsociety.org/voli5/iss I/artII/

Sousa Santos, B. de (20II). Producir para vivir: los caminos de la producción no capitalista. Estados Unidos: Fondo de Cultura Económica.

Tengö, M., Brondizio, E. S., Elmqvist, T., Malmer, P., y Spierenburg, M. (2014). Connecting diverse knowledge systems for enhanced ecosystem governance: The multiple evidence base approach. Ambio, 43(5), 579-59I. https://doi.org/10.1007/s13280-0I4-050I-3

Thompson, P. B. (2007). Agricultural sustainability: What it is and what it is not. International Journal of Agricultural Sustainability, 5(I), 5-16. https://doi.org/I0.1080/I4735903.2007.9684809

Tittonell, P. (2014a). Ecological intensification of agriculture-sustainable by nature. Current Opinion in Environmental Sustainability, 8, 53-6r. https://doi.org/10.1016/j.cosust.20I4.08.006

Tittonell, P. (20I4b). Livelihood strategies, resilience and transformability in African agroecosystems. Agricultural Systems, 126, 3-14. https://doi.org/10.1016/j.agsy.2013.10.010

Toledo, V. M., y Barrera-Bassols N. (2008). La memoria biocultural: importancia de las sabidurías tradicionales. Barcelona: Icaria.

Torres, L., Abraham, E. M., Rubio, C., Barbero-Sierra, C., y Ruiz-Pérez, M. (2015). Desertification research in Argentina. Land Degradation \& Development, 26(5), 433-440. https://doi.org/10.1002/ldr.2392

Turner II, B. L., Kasperson, R. E., Matson, P. A., McCarthy, J. J., Corell, R. W., Christensen, L., ... Schiller, A. (2003). A framework for vulnerability analysis in sustainability science. Proceedings of the National Academy of Sciences USA, I00(I4), 8074-8079. https://doi.org/I0.1073/pnas.1231335100

Vanclay, F., Howden, P., Mesiti, L., y Glyde, S. (2006). The social and intellectual construction of farming styles: Testing dutch ideas in australian agriculture. Sociologia Ruralis, 46(I), 6I-82. https://doi.org/I0.IIII/ j.1467-9523.2006.00404.x

Vasseur, C., Joannon, A., Aviron, S., Burel, F., Meynard, J. M., y Baudry, J. (2013). The cropping systems mosaic: How does the hidden heterogeneity of agricultural landscapes drive arthropod populations? Agriculture, Ecosystems \& Environment, 166, 3-14. https://doi.org/10.1016/j.agee.2012.08.013

Vélez Vargas, L. D. (2004). El paradigma científico de las ciencias agrarias: una reflexión. Revista Facultad Nacional de Agronomía Medellín, 57(I), 2145-2160. Recuperado de https://revistas.unal.edu.co/index.php/ refame/article/view/24139

Volante, J. N., Mosciaro, M. J., Morales Poclava, M. C., Vale, L. M., Castrillo, S. A., Sawchik, J., ... Paruelo, J. (2015). Expansión agrícola en Argentina, Bolivia, Paraguay, Uruguay y Chile entre 2000-2010: caracterización espacial mediante series temporales de índices de vegetación. Revista de Investigaciones Agropecuarias, 4I(2), 179-19r. Recuperado de http://ri.conicet.gov.ar/handle/11336/29589

Walker, B. H., Anderies, J. M., Kinzig, A. P., y Ryan, P. (2006). Exploring resilience in social-ecological systems through comparative studies and theory development: Introduction to the special issue. Ecology and Society, II(I), art. I2. Recuperado de https://www.ecologyandsociety.org/volis/issı/artı2/

Walker, B. H., Holling, C. S., Carpenter, S. R., y Kinzig, A. (2004). Resilience, adaptability and transformability in social-ecological systems. Ecology and Society, 9(2), art. 5. Recuperado de https://www.ecologyandsocie ty.org/vol9/iss2/art5/

Walker, B. H., y Salt, D. (2006). Resilience thinking. Sustaining ecosystems and people in a changing world. Washington: Island Press.

\section{Notas}

* Artículo de investigación 